\title{
Commentary: Keeping surgery relevant in oligometastatic non-small cell lung cancer
}

\author{
Jessica S. Donington, MD, MSCR
}

\author{
From the Section of Thoracic Surgery, University of Chicago Medicine and Biologic Sciences, Chicago, Ill. \\ Disclosures: J.S.D.: AstraZeneca: travel, honoraria, and advisory board. \\ Received for publication Oct 29, 2018; accepted for publication Oct 30, 2018; available ahead of print Dec 8, \\ 2018. \\ Address for reprints: Jessica S. Donington, MD, MSCR, University of Chicago Medicine, 5841 S. Maryland Ave, \\ MC 5040, Suite E-500, Chicago, IL 60637 (E-mail: jdonington@uchicago.edu). \\ J Thorac Cardiovasc Surg 2019;157:1629-30 \\ $0022-5223 / \$ 36.00$ \\ Copyright (C) 2018 by The American Association for Thoracic Surgery \\ https://doi.org/10.1016/j.jtcvs.2018.10.124
}

We are increasing our appreciation for the heterogeneity of stage IV non-small cell lung cancer (NSCLC). Improved systemic therapies are increasing survivals and the potential for benefit from local consolidative treatment. Surgery has been used in oligometastatic NSCLC for more than 40 years, with survival of $15 \%$ to $55 \%$, but broad adoption has been hampered by several factors: (1) All the supporting data are retrospective; (2) experience is limited to patients with a single metastatic site; and (3) attribution of the survival advantage to selection bias. There is currently excitement around recent prospective data supporting aggressive local therapy in oligometastatic NSCLC from radiation oncologists. The "Oligomez" trial is a small prospective phase II trial that closed early because of the significant progression-free survival improvement with the addition of local consolidative therapy to chemotherapy. ${ }^{1,2}$ Trial design was a key for success: randomizing patients with 3 or less metastases who did not progress on chemotherapy, thereby excluding patients with unfavorable tumor biology. Aggressive local therapy to all disease sites significantly improved progression-free survival (14.2 vs 4.4 months) and overall survival (41.2 vs 17.0 months). ${ }^{2}$ Surgery was part of local therapy in less than $25 \%$ of patients; the remainder received radiation (external beam or stereotactic body radiotherapy [SBRT]). Iyengar and colleagues ${ }^{3}$ reported similar results in another small prospective trial using only SBRT. These data have caught the attention of medical oncologists. There is potential for surgery to be overlooked in this evolution because of concern for the short-term morbidity compared with SBRT and speculation over the importance of the abscopal effect. ${ }^{4}$

To keep surgery relevant in oligometastatic NSCLC, beneficial evidence needs to go beyond small, singleinstitution retrospective series. This analysis of thoracic surgical interventions in advanced NSCLC from the National Cancer Database ${ }^{5}$ is an extension of work from the same authors in the California Cancer Registry. ${ }^{6}$ In each database,

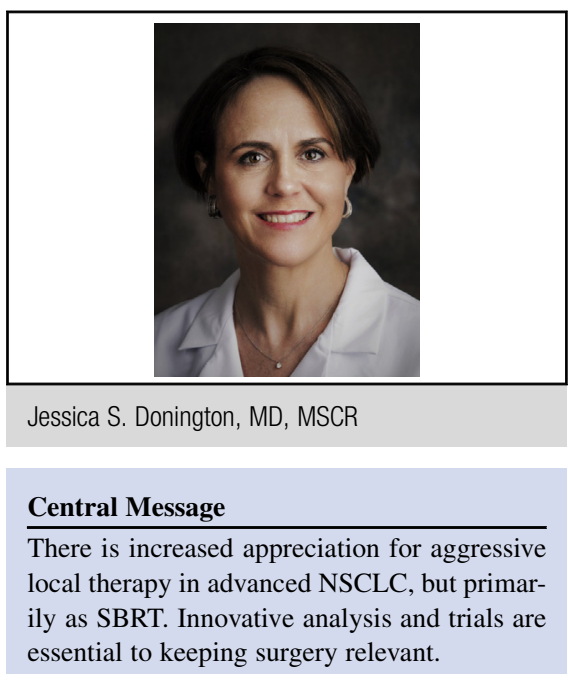

See Article page 1620.

they demonstrate improved survival with primary tumor resection. They now go 1 step further, creating a Surgical Selection Score (SSS) based on factors common to surgical patients: lower T, N, and M stage; age; and comorbidities. The SSS overcomes selection bias, fairly comparing retrospective cohorts, and could have prospective potential. Stage for stage, surgical patients have higher SSS scores, but when matched for SSS, adding surgery improves survival.

The increased appreciation for the use of local aggressive therapy in oligometastatic NSCLC patients should be applauded; but surgery should not be excluded from treatment plans. Surgery has a long history in this setting, but 2 small prospective SBRT trials are overshadowing 4 decades of retrospective surgical data. Cure is the goal for these patients; therefore, local control, improved staging, tissue acquisition for molecular analysis, and increased understanding of long-term toxicity afforded by resection validate its continued use. Prospective comparisons between surgical and nonsurgical therapies are challenging; therefore, insightful analyses are crucial. The SSS introduced by David and colleagues ${ }^{5}$ could serve as an innovative tool to select and stratify patients in prospective evaluations, which are essential to keeping surgery relevant in this setting.

\section{References}

1. Gomez DR, Blumenschein GR Jr, Lee JJ, Hernandez M, Ye R, Camidge DR, et al Local consolidative therapy versus maintenance therapy or observation for 
patients with oligometastatic non-small-cell lung cancer without progression after first-line systemic therapy: a multicentre, randomised, controlled, phase 2 study. Lancet Oncol. 2016;17:1672-82.

2. Gomez DR, Tang C, Zhang J, Blumenschein M, Hernandez JJ, Lee R, et al. Local consolidative therapy (LCT) improves overall survival (OS) compared to maintenance therapy/observation in oligometastatic non-small cell lung cancer (NSCLC): final results of a multicenter, randomized, controlled phase 2 trial. ASTRO Annual Meeting, San Antonio, Texas, Oct 21-24, 2018.

3. Iyengar P, Kavanagh BD, Wardak Z, Smith I, Ahn C, Gerber DE, et al. Phase II trial of stereotactic body radiation therapy combined with erlotinib for patients with limited but progressive metastatic non-small-cell lung cancer. J Clin Oncol. 2014;32:3824-30.

4. Bhalla N, Brooker R, Brada M. Combining immunotherapy and radiotherapy in lung cancer. J Thorac Dis. 2018;10:S1447-60.

5. David EA, Andersen SW, Beckett LA, Melinkow J, Clark JM, Brown LM, et al. Survival benefits associated with surgery for advanced non-small cell lung cancer. J Thorac Cardiovasc Surg. 2019;157:1620-8.

6. David EA, Canter RJ, Chen Y, Cooke DT, Cress RD. Surgical management of advanced non-small cell lung cancer is decreasing but is associated with improved survival. Ann Thorac Surg. 2016;102:1101-9. 